2 further adjusted for education and work status, model 3 included depression and self-rated health, and finally, model 4 included all covariates as well as physical activity, smoking and alcohol consumption. Additionally, interactions with age, sex, marital status, education, and work status were investigated.

Results The analytical sample had an average age of 65 years $(S D=7.01)$. We found that $22 \%$ of respondents engaged in gaming. Furthermore, of those participants who gamed, $65 \%$ were females and $48 \%$ were between 61 to 70 years old. Interaction analyses indicated that for younger individuals ( $\leq 65$ years), gaming predicted lower scores in the self-realization sub-scale of the CASP-19 $(\beta=-0.33,95 \%$ CI -0.62 to $0.04, p=0.03)$ in comparison to $>65+$ gamers. Furthermore, for widowed individuals, gaming predicted lower scores in CASP-19 $(\beta=-2.63,95 \%$ CI -4.68 - to $0.58, \mathrm{p}=0.01)$, indicating a lower quality of life in comparison to married, or single participants. Further analysis indicated that widowed individuals had lower scores in the self-realization $(\beta=-0.90$, $95 \% \mathrm{CI}-1.68$ to $-0.11, \mathrm{p}=0.03)$ and pleasure sub-scales $(\beta=-0.62,95 \%$ CI -1.17 - to $-0.07, p=0.03)$ of CASP- 19 . There was also a marginal association for widowed individuals who game and lower scores in the autonomy subscale $(\beta=-0.58,95 \% \mathrm{CI}-1.18$ to $0.12, \mathrm{p}=0.06)$. There were nonsignificant associations between gaming and loneliness and depression.

Conclusion Online gaming might be independently associated with lower levels of quality of life, particularly in terms of pleasure, self-realisation, and autonomy. This association might be modified by the individual's age and marital status. Widowed individuals who game appear to be more likely to report a lower sense of self-realisation, pleasure, and autonomy with some implications for their well-being.

\section{P73 USING PEER RESEARCH METHODS: TO EXPLORE HIGH MORTALITY RATES AMONG PEOPLE WITH MULTIPLE COMPLEX NEEDS}

${ }^{1}$ Emma A Adams*, ${ }^{1}$ Rachel Perry, ${ }^{1}$ Jill Harland, ${ }^{2}$ Angela Broadbridge, ${ }^{3}$ Emma L Giles, ${ }^{4}$ Grant J McGeechan, ${ }^{1}$ Amy O'Donnell, 'Sheena E Ramsay. ${ }^{1}$ Population Health Sciences Institute, Newcastle University, Newcastle upon Tyne, UK; ${ }^{2}$ Fulfilling Lives Newcastle Gateshead, Gateshead, UK; ${ }^{3}$ School of Health and Life Sciences, Teeside University, Middlesbrough, UK; ${ }^{4}$ Centre for Applied Psychological Science, Teeside University, Middlesbrough, UK

\subsection{6/jech-2021-SSMabstracts. 161}

Background Multiple complex needs ( $\mathrm{MCN}$ ) is one of a number of terms used to describe people facing co-occurring issues of homelessness, substance misuse, offending, and mental illhealth. When considered in isolation, homelessness, substance misuse, mental ill-health and offending, each contribute to high mortality rates; however, when co-occurring this effect is amplified. A large amount of the mortality research to date within MCN populations has been epidemiological. Despite acknowledge of the benefits of conducting research withindividuals rather than on, there is limited involvement of peer research methods in current studies exploring mortality and MCN. This research aimed to explore the perspectives of people with lived experience of MCN and professionals who support them on the perceived reasons underlying high mortality rates among people with $\mathrm{MCN}$.

Methods Using peer research methods, three focus group discussions (21 participants) were conducted within the North
East of England to explore the perspectives of people with $\mathrm{MCN}$, frontline staff, and managers/commissioners.

Results Findings from this study provide valuable perspectives of people with $\mathrm{MCN}$ and those that provide them with support on what may be perceived factors underlying premature mortality. Mental ill-health and substance misuse (often cooccurring dual diagnosis) were perceived as influencing premature mortality among MCN groups. Perceptions of opportunities for identify people at-risk included critical life events (e.g. bereavement, relationship breakdown) and transitions (e.g. release from prison, completion of drug treatment). Early prevention, particularly supporting young people experiencing adverse childhood experiences was also highlighted as a priority.

Conclusion High mortality in MCN groups may be reduced by addressing dual diagnosis, providing more support at critical life events, and investing in early prevention efforts. The novelty of this study is it has shown the value of listening to and conducting research with individuals with experience of MCN. It enabled an exploration of an issue that directly affects their community and supported understanding of the personal perspective of a handful of people with MCN and their carers on what may have informed premature mortality among their networks, as well as identifying perceived avenues for possible preventive interventions within the local region. Furthermore, the insights specific to opportunities for service provision take into consideration the intricate nature of $\mathrm{MCN}$ and improve service access and navigation.

\section{P74 A LIFECOURSE PERSPECTIVE ON HISTORICAL DEMOGRAPHIC PATTERNS OF CIRCULATORY SYSTEM DISEASE AND ALL-CAUSE MORTALITY IN TWENTIETH CENTURY AUSTRALIA}

${ }^{1}$ Cecily C Kelleher*, ${ }^{2}$ Gabrielle Kelly, ${ }^{1}$ Ricardo Segurado, ${ }^{1,3}$ Jonathan Briody, ${ }^{4}$ Alexander M Sellers, ${ }^{5}$ Janet McCalman. ${ }^{1}$ School of Public Health, Physiotherapy and Sports Science, University College Dublin, Dublin, Ireland; ' ${ }^{S}$ chool of Mathematics and Statistics, University College Dublin, Dublin, Ireland; ${ }^{3}$ School of Economics, University College Dublin, Dublin, Ireland; ${ }^{4}$ Department of Medicine, Royal Adelaide Hospital, Adelaide, Australia; ${ }^{5}$ School of Population and Global Health, University of Melbourne, Melbourne, Australia

\subsection{6/jech-2021-SSMabstracts. 162}

Background Circulatory System Disease (CSD) patterns vary over time and between countries, related to lifestyle risk factors across the life course, associated in turn with socioeconomic circumstances. Current global CSD epidemics in developing economies are similar in scale to those observed previously in the United States and Australia. We examine from a lifecourse perspective the historical context in Australia for the rise and decline of CSD based on the published scientific literature and population trends. Past epidemiological studies focused on the relative advantage of those of Southern European compared to those of British or Irish origin. Historical retrospective cohort studies in populations including world war one veterans and maternity hospital data have shown early life influences on later health outcomes.

Methods We employed data from census-derived place of birth by age bracket and sex from 1891 to 1986, based on digitised paper record. CSD mortality rates were available from 1907 to 2016 and age-specific rates were computed for the general population. All-cause mortality for the foreignborn (fb) from 1910 to 1980 was not readily available, as 
country of origin was not recorded at death. It was constructed based on numbers of foreign-born in specific agegroups at each census employing a novel actuarial method. For example, if $\mathrm{p}$ is the mortality rate for the $\mathrm{fb}$ age 30 per year in the interval 1947-1954 then $\mathrm{p}$ is estimated from the data by 1- (number all fb age 35-45 in 1954/number all $\mathrm{fb}$ age $25-35$ in 1947$)^{1 / 7}$. $p$ for other age-groups is calculated similarly. For all age-groups $\mathrm{p}$ will underestimate the mortality rate.

Results The Australian population grew exponentially between 1861 and 1986, reaching 15,602,150 million; its demographic composition was $64.7 \%$ foreign born initially and of predominantly British and Irish origin. Later twentieth century immigration was from mainly Southern European and Asian countries and FB accounted for a smaller proportion (22.4\%) of the population by 1986. A strong period effect across all age groups and both genders was observed for CSD mortality. Rates of CSD rose consistently, particularly from the 1940 s onwards, peaked in the 1960 s and then began to decline sharply in the 1980s. Both male and female foreign-born showed similar all-cause mortality patterns at age midpoints from 30 through to 60 years of age.

Conclusion Our findings support both a commonly experienced period effect for CSD mortality but suggest also that early origins of foreign-born may contribute to mortality patterns.

\section{P75 CONTEXTUAL INFLUENCES ON CHRONIC DISEASE: A MULTI-LEVEL ANALYSIS IN THE TWIN CITY OF RAMALLAH AND AL BIREH IN THE OCCUPIED PALESTINIAN TERRITORY}

Ahmad Alkhatib*, Jonathan Olsen, Richard Mitchell. MRC/CSO Social and Public Health Sciences Unit, University of Glasgow, Glasgow, UK

\subsection{6/jech-2021-SSMabstracts. 163}

Background The features of the urban environment can support human health as well as harm it, but less is known about the context of middle eastern countries. The association between green space and the political classification of the urban environment and the risk of chronic illness was investigated in a novel setting, the twin city of Ramallah and Albireh in the occupied Palestinian territory.

Methods We used multi-level regression analysis to link the 2017 census data with contextual characteristics from Geographic Information System data. The outcome variable is the presence or absence of chronic illness. The contextual explanatory variables are: the proportion of three types of green space in the areas of residence (mixed trees, crop trees and open space with little or no vegetation); the locality of residence (Ramallah, Albireh and Refugee camps); and political land classification (Area A, B, and C). Generalised multi-level analysis was conducted in $\mathrm{R}$ using 'lme4' package, with individuals at level one $(n=54693)$ and areas of residence at level two $(n=228)$, adjusting for individual demographic and socioeconomic characteristics.

Results Just over $13 \%$ of the chronic illness risk variation was attributed to the area of residence. On the political dimension and while accounting for the individual-level factors, only living in the context of a refugee camp had a significant positive association (OR 1.91 CI [1.17-3.09]). However, the association between refugee camps and chronic illness rendered non-significant when green space variables were entered into the model. In the final model, only the proportions of 'mixed' tress in the residential areas had a significant inverse association with the risk of chronic illness (OR $0.96 \mathrm{CI}$ [0.95-0.97]), all else being equal.

Conclusion Within the confines of a cross-sectional, observational study, the findings shed light on the possible role of the urban environment context as a determinant of health, irrespective of individual-level factors. Our ability to differentiate between several types of green space was important, as findings demonstrated that not all types are associated with reduced risk of chronic illness. Our results from a middle eastern setting add to the existing evidence, largely in Western countries, that trees in urban settings are important and beneficial to human health. Also, to reduce inequalities in health researchers and policymakers should give more attention to the disadvantaged contexts of refugee camps.

\section{P76 INCIDENCE, OUTCOMES AND CHARACTERISTICS OF OUT-OF-HOSPITAL CARDIAC ARRESTS IN PATIENTS WITH PSYCHIATRIC ILLNESS: A SYSTEMATIC REVIEW}

${ }^{1}$ Raied Alotaibi* ${ }^{1}$ 'Laura Bijman, ${ }^{1}$ Nynke Halbesma, ${ }^{2}$ Gareth Clegg, ${ }^{1}$ Caroline Jackson. 'Usher Institute, University of Edinburgh, Edinburgh, UK; ${ }^{2}$ Resuscitation Research Group, University of Edinburgh, Edinburgh, UK

\subsection{6/jech-2021-SSMabstracts. 164}

Background People with a history of psychiatric illness have a lower life expectancy than people without psychiatric illness, largely due to an excess burden of cardiovascular disease (CVD). Despite the well-established association between psychiatric illnesses and risk of CVD in general, little is known about psychiatric illness relates to the incidence and outcomes of out-of-hospital cardiac arrest (OHCA) specifically. We therefore aimed to conduct a systematic review of the literature to critically appraise and summarise the existing evidence on incidence, outcomes and characteristics of OHCA in patients with psychiatric illness.

Methods We searched Embase, Medline, PsycINFO and Web of Science from the first publication within each database to 16th December 2020 using a detailed electronic search strategy containing a wide range of terms for psychiatric illness and OHCA. We included observational studies that reported on the characteristics of patients with OHCA or OHCA incidence or survival by psychiatric illness status (or that reported on patients with psychiatric illness without including a comparison group). Two authors independently screened the search results, assessed risk of bias in relevant studies and extracted data. We registered the protocol of this review with PROSPERO (CRD42021229545).

Results Our search retrieved 10,610 potentially eligible studies, of which nine met our inclusion criteria. Of these, eight were retrospective cohort studies and one was a case-control study comprising 5,906 OHCA patients with history of psychiatric illness across five countries. Three studies included patients with depression only, whilst six studies varied in their definition of psychiatric illness. Eight studies reported on psychiatric illness status with respect to OHCA incidence and only one study assessed OHCA outcome. Most studies found that psychiatric illness was associated with an increased risk of OHCA. Among patients with an OHCA, those with 